\title{
Historical Supernovae and Supernova Remnants
}

\author{
By ZHENRU WA NG \\ Center of Astronomy and Astrophysics, CCAST World Laboratory \\ and Department of Astronomy, Nanjing University, Nanjing, China $\dagger$
}

The oldest historical supernova (SN), recorded by ancient Chinese in 14th Century B.C. on pieces of tortoise shells or bones, is identified with the aid of modern space $\gamma$-ray observations. Hard X-rays with energy up to $20 \mathrm{keV}$ were observed from IC 443 by the X-ray satellite Ginga. We infer from these observations the age of IC 443 is $\sim 1000-1400$ yrs. The result supports the hypothesis that IC 443 is the remnant of the historical SN 837 that occurred during the Tang Dynasty.

The association between the supernova remnant (SNR) CTB 80 and SN 1408 has been hotly debated for about ten years and is briefly reviewed and discussed here. A new picture is presented to explain this association.

High energy emission from historical SNRs can persist in a multiphase interstellar medium (ISM). As a result, the study of the relationship between SNRs and ancient guest stars has gained new vitality.

\section{The First Supernova Observed by Mankind}

SN 1987A, the first supernova observed by the naked eye in nearly 400 years, stimulates a high tide in supernova research. It also tempts us to ask: what is the earliest supernova recorded by mankind? Recently, we have discussed this topic in a few articles (Wang $1987 \mathrm{a}, \mathrm{b} ; \mathrm{Xu}$, Wang \& Qu 1992). The earliest supernova recorded by mankind is the great new star that occurred in 14th century B.C. recorded by the ancient Chinese on a piece of Tortoise shell or bone in Yin-Shang Dynasty (Fig. 1). The text of the inscription is shown in Fig. 2.

According to the above inscription, two conclusions can be drawn: (1) The ancient event was described as a great new star instead of a new star. This indicates that it should be very bright - bright enough to be considered as a nearby supernova. (2) Its visual position is near Antares ( $\alpha$ Sco).

Searching within one degree of $\alpha$ Sco, we find the $\gamma$-ray source 2CG 353+16 detected by the European Satellite COS B (Swanenburg et al. 1981). On the basis of the above observed facts, I proposed that 2CG $353+16$ might be the compact remnant of the great new star that occurred in the 14th Century B.C. (Wang 1987a, b). The main reason is that neutron stars can be formed in Type II supernova explosions and some wellknown young neutron stars, such as Crab and Vela pulsars, have strong and stable $\gamma$-ray emission.

As shown by Mayer-Hasselwander (1980), the position of $2 \mathrm{GC} 353+16$ is coincident with that of the $\rho$ Oph cloud. Assuming that it is a supernova with maximum absolute magnitude $M_{V}=-18^{m} \pm 2.5$ (Trimble 1982) that exploded in the $\rho$ Oph cloud, at a distance $D=160 \mathrm{pc}$ (Encrenaz et al. 1975; Whetted 1974) and visual extinction $A_{V}=7.1$ (Lose 1986), we estimate that it should have visual magnitude $m_{V}=M_{V}+$ $A_{V}-5-5 \log D=-4.9 \pm 2.5$, which could be easily observed by the ancients.

$\dagger$ Project supported by the National Science Foundation of China and the State Education Commission of China 


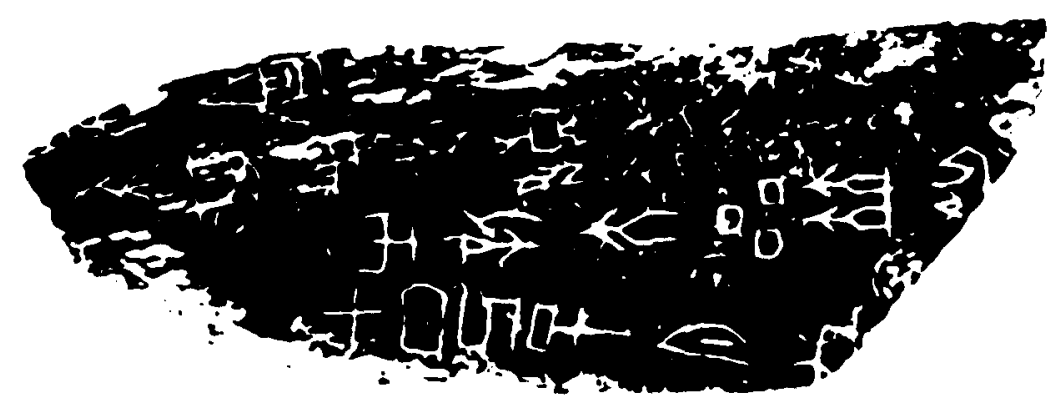

Figure 1. An Ancient Chinese Oracle Bone with Inscription about a Great New Star that Occurred in the 14th Century B.C.

$$
\begin{aligned}
& \text { 七日已己夕总出新大星并火 } \\
& \text { 刋墟上契后㷁 } \\
& \text { 下.九.二 }
\end{aligned}
$$

FIgure 2. Chinese record of the appearance of the great new star observed in the Yin-Shang Dynasty. "On the 7th day of the month, a Ji-Si day, a great new star appears in company with Antares ( $\alpha \mathrm{Sco}$ )." [From the second part of the ninth chapter of the book Yin-Xu-Shu-Qi-Hou-Bian]

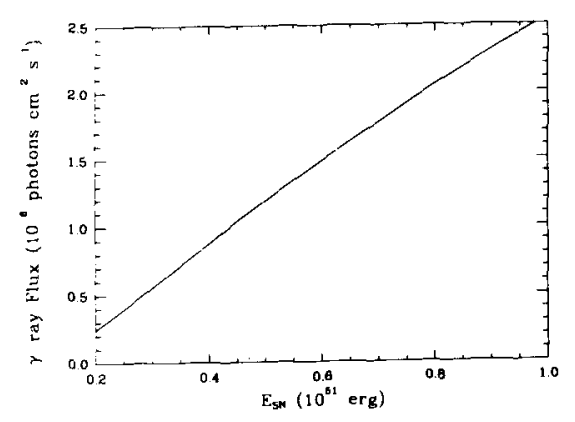

FIGURE 3. The relation between the $\gamma$-ray flux and the kinetic energy of the supernova explosion that occurred in the 14th century B.C. 


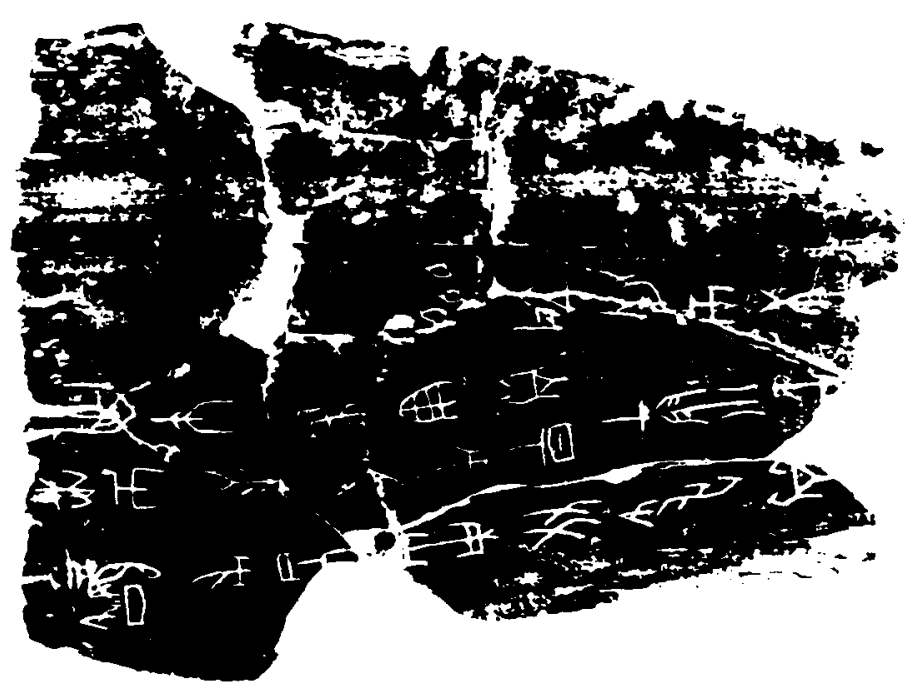

Figure 4. Another oracle bone with inscription about a new star

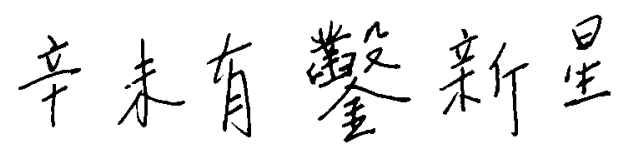

Figure 5. Text of the inscriptions on bones or tortoise shells related to Fig. 3

$\gamma$-ray emission follows the decay of $\pi_{0}$ mesons produced from the interaction between atomic nuclei in the cloud and high energy protons in cosmic rays. Assuming that the observed $\gamma$-ray flux of 2 GC $353+16$ is due to this mechanism, Black \& Fazio (1973) estimated that the mass of the $\rho$ Oph cloud is about five times larger than that implied from its radio observation (Montmerle et al. 1983). (Hiller 1984) pointed out the difficulties in accounting for the $\gamma$-ray flux of $2 \mathrm{GC} 353+16$ as a result of cosmic ray interactions in the $\rho$ Oph cloud.

Recently we proposed (Xu, Wang, \& Qu 1992) that the $\gamma$-ray flux of 2GC $353+16$ may result from the interaction between protons in the molecular cloud and the high energy protons that originate from a supernova that exploded in a pre-supernova stellar wind cavity within the $\rho$ Oph cloud. The calculated $\gamma$-ray photon flux is comparable with the observed one, $(1.1 \pm 0.4) 10^{-6} \mathrm{~cm}^{-2} \mathrm{~s}^{-1}$ (Fig. 3).

There are some other inscriptions about new stars or great stars on bones or tortoise shells from the Yin-Shang Dynasty. The most certain one among them is shown in Fig. 4 (Guo 1980). The text of its inscription is shown in Fig. 5. Some people considered it to be a record of the same event of Fig. 1. Since no position information is available in Fig. 4 or 5 , it is difficult to say any more about it. 


\section{SNR IC 443 and the guest star AD 837 that occurred during the Tang Dynasty}

IC 443 (G189.1+30, 3C157) has been studied extensively in radio, infrared, optical and $\mathrm{X}$-rays. These studies show that there is a radio, optical and soft $\mathrm{X}$-ray enhancement in its NE quadrant and a radio and optical shell with a diameter of about 40' (Petre et al. 1988). The NE enhancement is generally believed to be the result of an encounter between the shock and a dense HI cloud (De Noyer 1978). The distance of IC 443 is considered to be about $1.5 \mathrm{kpc}$ (Fesen 1984). Strong infrared emission from IC 443, with luminosity $\sim 10^{37} \mathrm{ergs}^{-1}$, was observed by IRAS (Mufson et al. 1986; Braun \& Strom 1986; Dwek et al. 1987).

Hard X-rays from IC 443, with energies up to $20 \mathrm{keV}$, were observed by Ginga (Wang et al. 1992a). The X-ray spectrum of IC 443 can be fitted by non-equilibrium model of Masai (1984) with two thermal components, i.e., $k T_{s}=0.9 \mathrm{keV}$ for the soft component and $k T_{h}=14.3 \mathrm{keV}$ for the hard component, as shown in Fig. 6. The IPC map (Petre et al. 1988), shows that the soft X-rays come mainly from the NE quadrant. Subtracting this component, we can fit the spectrum of the hard component. The allowed region in the $T_{h}-T_{s}$ plane is shown in Fig. 7 .

It is natural that the $\mathrm{X}$-rays are brighter from areas with higher number density and lower temperature. This is just the case of the brighter NE quadrant of the IPC map of IC 443. Hence the hard X-ray emission should come from its SW and the W regions, i.e., those areas in the IPC map with lower surface brightness of soft X-rays. This conclusion is consistent with the locations of the error boxes of HED3 (2-60 keV) and MED (2-40 $\mathrm{keV}$ ) indicated by the HEAO $1 \mathrm{~A}-2$ observation and the IPC hardness ratio map (Petre et al. 1988). It is also consistent with the morphology of the multiwavelength map. The curvature radius of the NE area is smaller than the curvature radius of the SW area (Petre et al. 1988). This result is consistent with the idea that the SW quadrant has lower density, hence higher shock velocity and a larger curvature radius. We expect that future hard X-ray images from AXAF and ASTRO D will verify the above conclusion.

In a multiphase ISM, supernova explosions will give rise to various morphologies of SNRs. In general, we may expect the velocity of the blast wave to vary substantially in different parts of such a SNR. The morphology of CTB 109 is a wonderful example of the interaction between a supernova explosion and a molecular cloud, which has resulted in a semicircular shell (Wang et al. 1992b).

The multi-temperature model of IC 443 indicates an inhomogeneity in density. With a shock velocity corresponding to the hard component as high as $2.5-3.5 \times 10^{3} \mathrm{~km} \mathrm{~s}^{-1}$, we can estimate shock crossing time to be 1000 - 1400 years. If so, the supernova should have occurred in historical times and its outburst may have been recorded by careful observers.

Shklovsky (1954) and Shajn \& Gaze (1954a,b) suggested that IC 443 could be the remnant of an ancient guest star recorded in $\mathrm{AD} 837$, because of its positional coincidence. However, Xi (1955) argued against this identification and suggested that the "guest star" was in fact Comet Halley. Moreover, the idea that IC 443 is an old SNR was supported by pre-Ginga observations. Now, however, the hard component observed by Ginga implies that the age of IC 443 is $1000-1400$ years, which seems consistent with the idea of IC 443 being the remnant of the guest star AD 837. Thus it is imperative to investigate the Chinese records of this event.

Several records of a guest star are found in Xin-Tang-Shu (Ouyang and Song 1061) and Weng-Xian-Tong-Kao (Ma 1254). Let us first note the following record for the guest star: "on a Jia-Shen day (April 29) in the 3rd month of the 2nd year of the Tang-Wen-Zong 

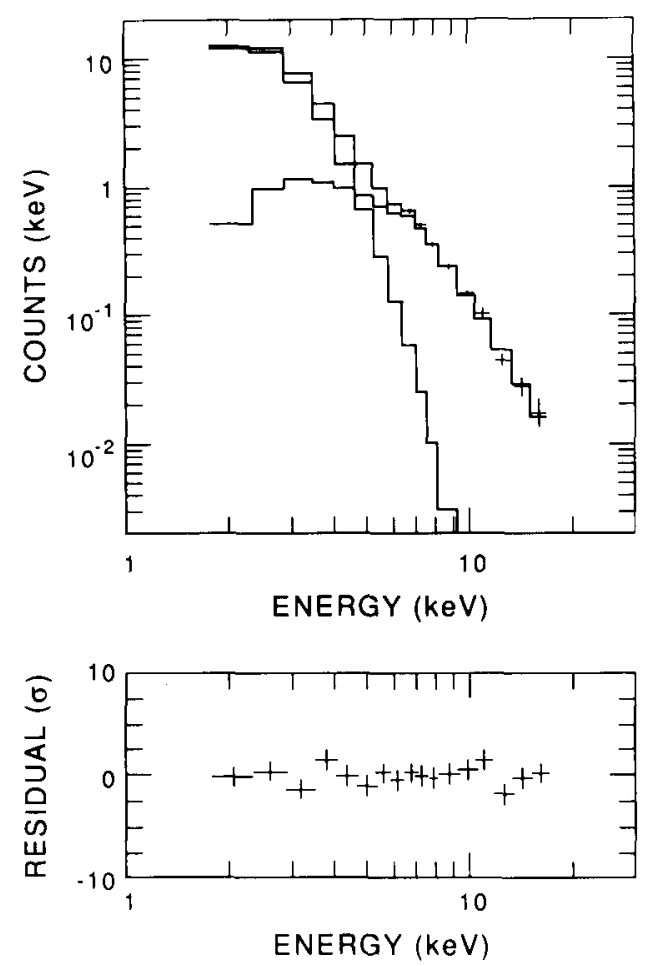

FIgURE 6 . The observed spectrum and its best fit with two thermal components $T_{h}=14.3$ $\mathrm{keV}$ and $T_{s}=0.9 \mathrm{keV}$

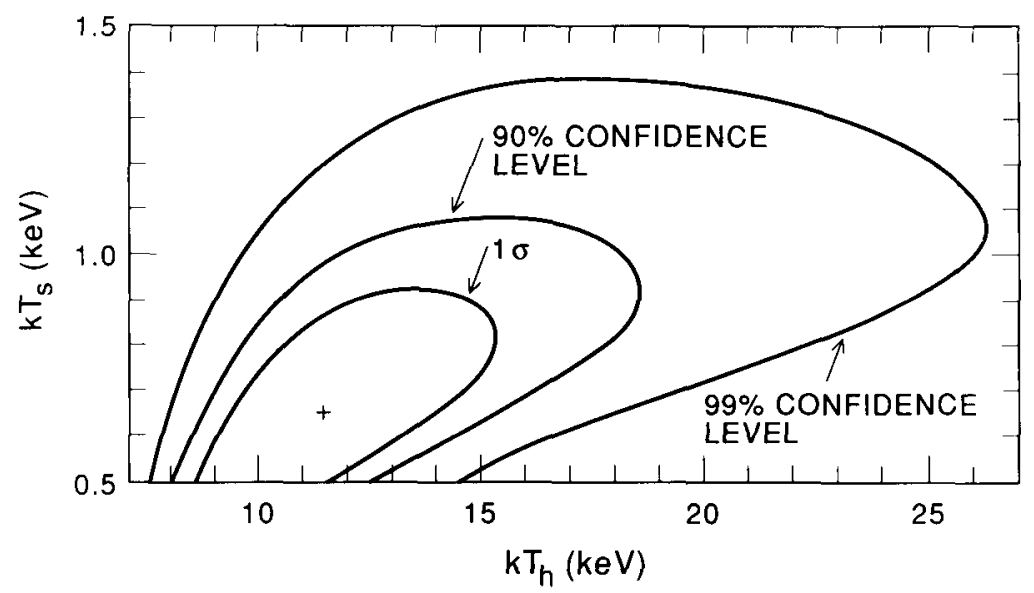

FIGURE 7. The allowed regions of electron of temperature for the two component thermal bremsstrahlung model

Kai-Cheng reign-period AD 837), a guest star appeared below the Dong-Jing (Gem., 22nd lunar mansion). On a Bing-Wu day in the fourth month (May 21), the guest star below the Dong-Jing went out of sight." (Ouyang and Song, 1061; Ma, 1254 ). See Figs. 8 and 9.

The guest star of AD 837 is included in the supernova catalogues of Biot (1846), Williams (1871), Lundmark (1921), Ho (1962) and Xi \& Bo (1965). Some records are 


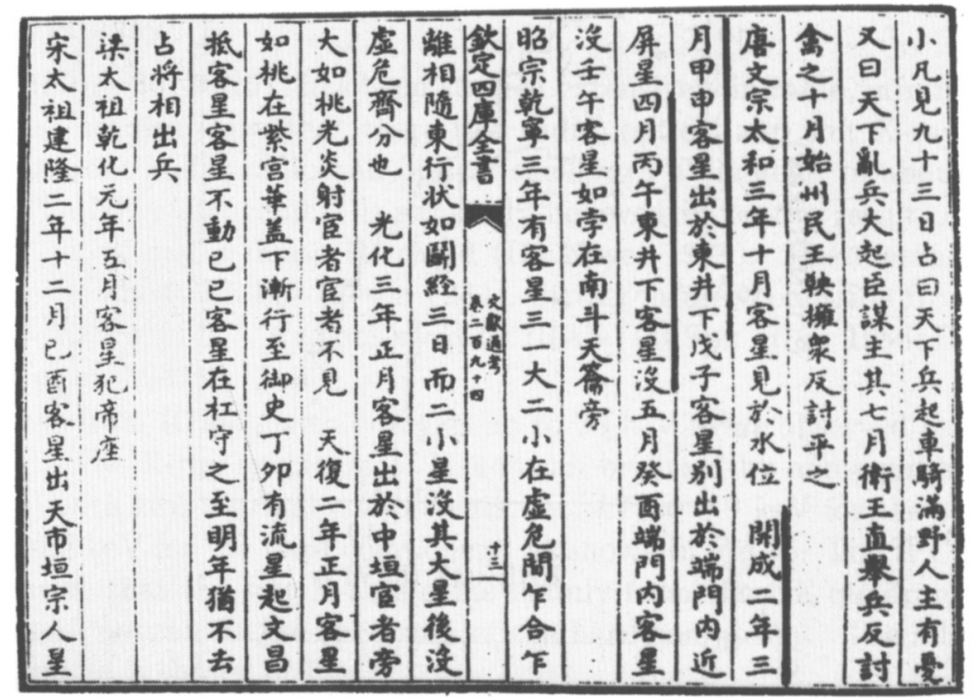

FIgURE 8. A copy of the ancient record in Wen-Xian-Tong-Kao for SN 837

also found for Comet Halley, for example, the following comment in Xin-Tang-Shu: "On a Gui-wei day (April 28), it was about $3 \mathrm{ft}$ long. It then disappeared at the right of Xuan Yuan (Leo). It is normal for a comet to point westward in the morning and eastward in the evening." (Ouyang and Song 1061). See Fig. 9.

Clearly, it is not possible for Comet Halley to move from Leo on 28 April to Gemini on 29 April, i.e., a distance of $45^{\circ}$ in one day (Xi \& Bo 1965), and then to stay at a fixed position in Gemini for 3 weeks. We believe that the records of AD 837 must refer to two separate events, one being a supernova and the other being Comet Halley. Because IC 433 has an age $1000-1400$ years derived from the hard X-ray observations and a position in agreement with that of the guest star of $\mathrm{AD} \mathrm{837,} \mathrm{we} \mathrm{conclude} \mathrm{that} \mathrm{it} \mathrm{is} \mathrm{the}$ remnant of that event, the historical records of which are reproduced in Figures 8 and 9.

\section{CTB 80 and the Historical Record of AD 1408}

CTB 80 is a very fascinating object. Its nature has been hotly debated for more than ten years. Strom, Angerhofer \& Velusamy (1980), Strom, Angerhofer \& Dickel (1984), and Wang \& Seward (1984) suggested that it is the remnant of SN 1408. However, people have become skeptical about this identification because the pulsar $1951+32$ that was discovered within CTB 80 has a characteristic spin-down age $\sim 10^{5}$ yrs (Kulkarni et al. 1988). The detection of the infrared and HI shells around CTB 80 of diameter nearly $1^{\circ}$ (Fesen et al. 1988, Koo et al. 1990) increased the suspicion that the AD 1408 event was a supernova (Stephenson \& Yau 1986). Thus, the suggested association between CTB 80 and the AD 1408 has many difficulties. Let us discuss them one by one.

We first consider the historical records of the $\mathrm{AD} 1408$ event. There are many such records. Some of them have been well introduced in recent papers and books (Li 1979, 1987; Wang \& Seward 1984; Zhuang et al. 1987). Here we just mention a typical one, written in Ming Taizong Shilu (essential records of the Ming Dynasty Emperor Taizong) as shown in Fig. 10. The text of Fig. 10 can be translated as follows:

"On Oct. 24, 1408, at night, near the meridian, to the southeast of Niandao (along 


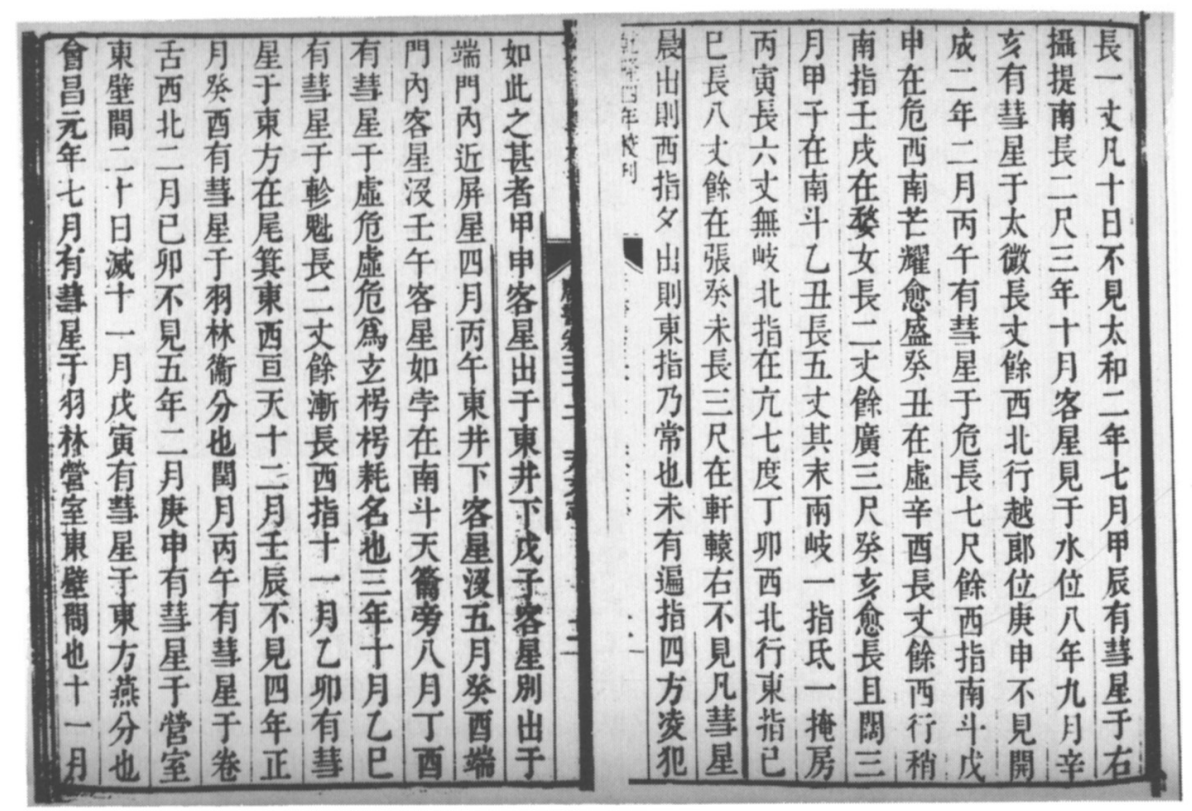

Figure 9. A copy of the ancient record in Wen-Xian-Tong-Kao. Left - record for SN 837; right - record for Comet Halley

the line from $13, \eta, \theta$ Lyr to $4,17 \mathrm{Cyg}$, Fig. 11), there is a star like a lamp, its color is yellow and its lustre smooth, it shows up and does not move, it is a Zhou-bo, a virtuous star."

Similar texts were recorded during Sept. 10 to Oct. 24, 1408 in China. There are also Japanese records (Imaeda \& Kiang 1980; Ho 1962; Xi 1982). The first Japanese record was dated July 14, 1408, 58 days earlier than the first Chinese record.

The most important key word in the records of the AD 1408 event is "Zhou-bo," a very unusual reference to its "nobility" or brightness. This word has been used to describe a guest star on only one other occasion - SN 1006, the brightest recorded supernova in history (see History of Song Dynasty, Astron. Chap. 9, P1226).

Another key phrase is "like a lamp." The only other known use of this phrase in astronomical records was in the historical record of SN 1572 in the Essential Records of the Ming Dynasty Emperor Shenzong. The key phrase indicating the position is the reference to the southwest of Niandao, exactly the location of CTB 80 (Fig. 11).

Summarizing the analyses of the above key words, we have little doubt that the event of AD 1408 was a very bright historical supernova located exactly at the center of CTB 80 . This is the most compelling evidence for our conclusion.

Now let us discuss the age of PSR 1951+32. Pulsars are thought to spin down according to the law

$$
P^{2}-P_{o}^{2}=2 P \dot{P} t
$$

where $P$ and $P_{o}$ are the period of a pulsar at the present time and at its birth time respectively. When $P \gg P_{o}$, we can infer the age from the characteristic spin-down time

$$
t=\tau_{c}=P / 2 \dot{P}
$$

where $\tau_{c}$ is the characteristic age of a pulsar. But otherwise, the age of a pulsar is given 


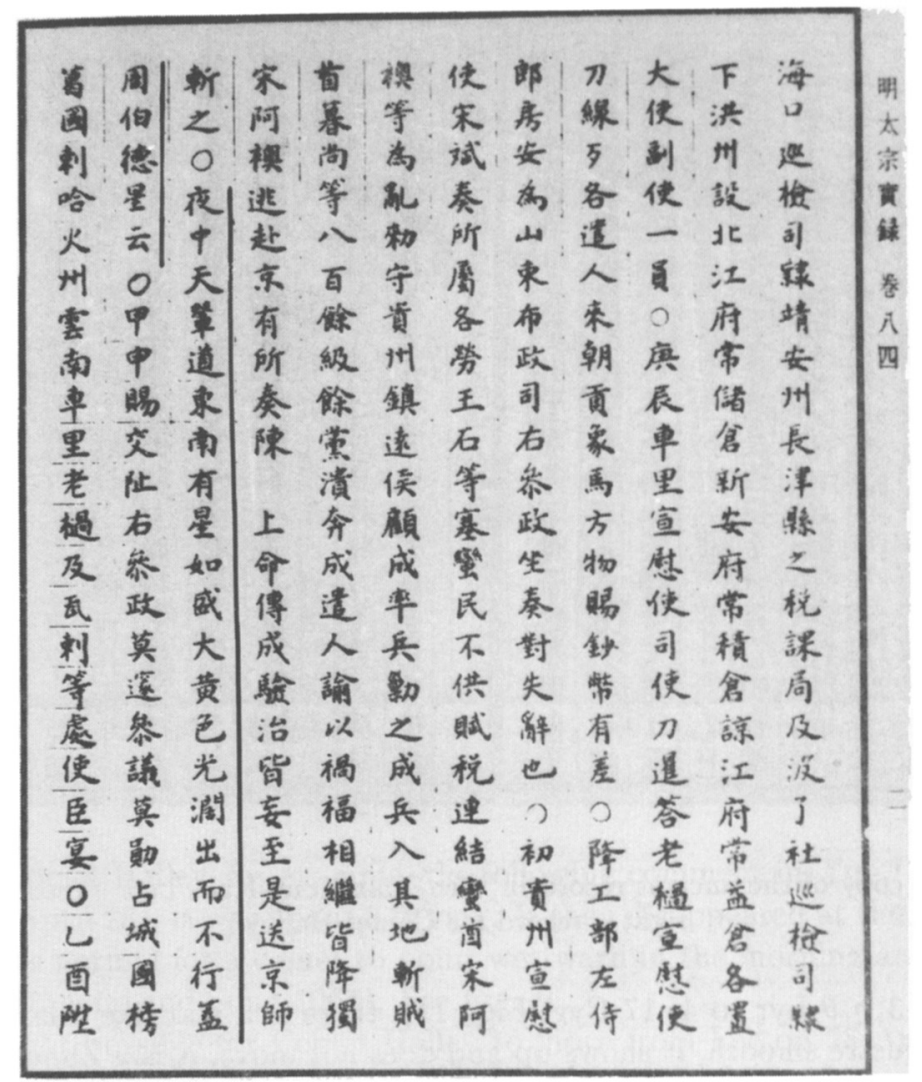

Figure 10. A copy of the ancient record for SN 1408 .

by

$$
t=\frac{P^{2}-P_{o}^{2}}{2 P \dot{P}}
$$

Equation (3) shows that PSR 1951+32 is not necessarily as old as its spin-down time. It can be a young pulsar with age of 585 yrs if its initial period was $P_{0}=0.0394 \mathrm{~s}$. If so, there is no difficulty in assuming that PSR $1951+32$ was formed in AD 1408.

Since PSR $1951+32$ is the central source of CTB 80, the central part of CTB 80 should be the remnant of a Type II supernova explosion. Probably, the IR/HI shell that surrounds CTB 80 was formed some $10^{5}$ yrs earlier by the explosion of another supernova in the same association - perhaps a binary companion of the progenitor of PSR $1951+32$. Since then the progenitor of PSR $1951+32$ could have moved to the west at a velocity $\sim 200 \mathrm{~km} \mathrm{~s}^{-1}$. The low velocity, high density filaments outside the central core of CTB 80 may be circumstellar matter ejected by the progenitor of PSR $1931+32$ itself during its red giant stage. More quantitative work is needed to develop this new picture of the association between CTB 80 and SN 1408 . 


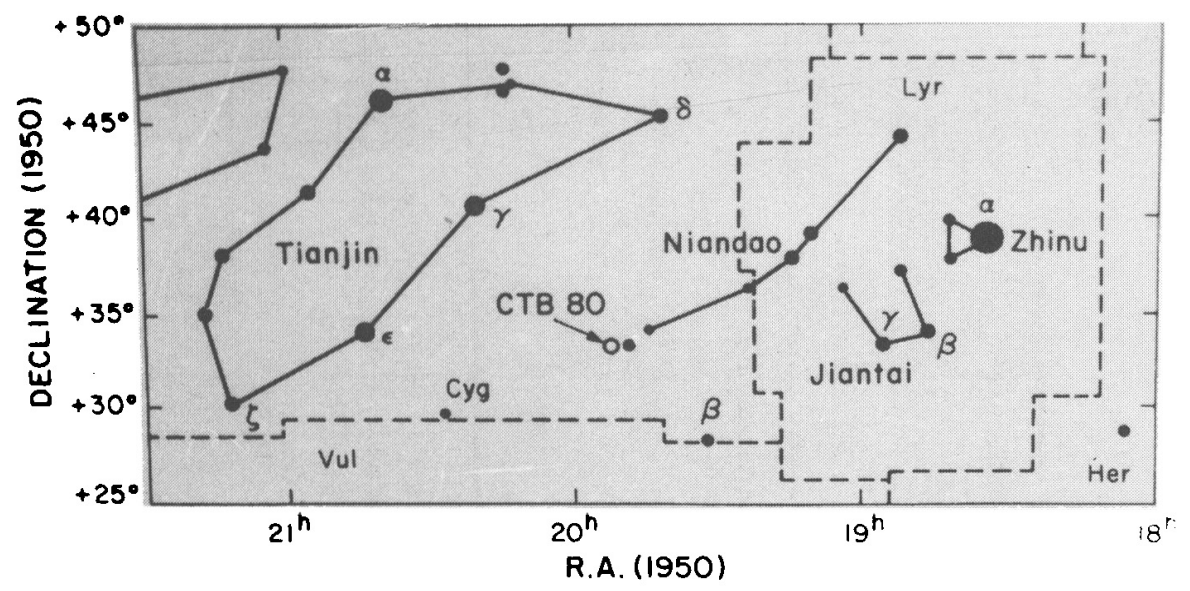

Figure 11. The position of CTB 80 related to Niandao

\section{Conclusions}

The most certain identifications between SNR and historical supernovae are as follows (Clark \& Stephenson 1977):

$$
\begin{array}{ll}
\text { G184.6-5.8(Crab) } & \text { SN 1054 } \\
\text { G327.6+14.5 } & \text { SN 1006 } \\
\text { G120.1+1.4 } & \text { SN 1572 } \\
\text { G4.5+6.8 } & \text { SN 1604 }
\end{array}
$$

Some other probable, possible, and tentative identifications have been proposed by Shklovsky (1954) Shajn \& Gaze(1954a,b), Xi \& Bo (1955), Clark \& Stephenson (1977), Li (1979), Strom et al. (1980), Wang et al. (1986) and Wang (1987a,b).

Before the 1980's, the identifications between SNRs and historical supernovae were based mainly on positional coincidences and estimates of the age of SNRs deduced from radio and optical observations. Moreover, the model of a uniform ISM was taken for granted. Such identifications easily gained acceptance. Now, we have begun to pay more attention, on the one hand to the X-ray and $\gamma$-ray observations, and on the other hand to the possibility that supernova progenitors are embedded in a multiphase medium. The possibilities include a molecular cloud, matter ejected in a stellar wind, the cavity due to a previous supernova, or a wind-driven bubble. As we have discussed, these scenarios give new views to the problem of determining the ages of some SNRs.

We expect that future observations of images of SNRs in hard X-rays and $\gamma$-rays will yield some exciting results to the age of SNR.s. Theoretical and observational research on the nonuniform ISM and its effect on the evolution of SNRs will provide new paths to determining the ages of some SNRs. The task to identify SNRs with historical supernovae is far from complete, but continues with rosy prospects.

\section{REFERENCES}

Biot, M. E. 1846, Connais Temps, Additions, 60

Black, J. H. \& Fazio, G. G. 1973, Ap. J., 185, L7

Braun, R. \& Strom, R. G. 1986, A. Ap. 164,193

Clark, D. H. \& Stephenson, F. R. 1977, The Historical Supernovae (Oxford: Pergamon Press) DeNoyer, L. K. 1978 , M.N.R.A.S., 183, 18

Dwek, E., Petre, R., Szymkowiak, A., \& Rice, W. 1987, Ap. J. 320, L27 